\title{
Literarisierter Migrationsbegriff und Balkanmimikry als europäische Herausforderung für die Komparatistik
}

Aglaia Blioumi

\begin{abstract}
This paper compares common narrative and writing strategies in Marica Bodrožić's German novel Sterne erben, Sterne färben. Meine Ankunft in Wörtern von (2007) and

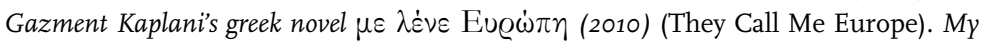
thesis is that the perception of the narrative theme of modern migration creates common narrative and writing strategies leading to a smigratory memory of European literaturer. On the level of genre the paper focuses properly on the autobiographical consistency of the texts, while on the level of the content on the significance of modern migration, the reminiscences of Communism in former Serbia and Albania and finally the Balkan and Balkanism as literary construction.
\end{abstract}

Title: Literarized Concept of Migration and Balkan Mimicry as a European Challenge for Comparative Literature

Keywords: migration literature; European literature; Communism; Balkan

Derzeit gilt >Migrationsliteratur < als eine sowohl auf dem Buchmarkt erfolgversprechende (vgl. Krauze-Olejniczak 2016: 113) wie auch literaturwissenschaftlich anerkannte Literatur, zumal man »nicht mehr außerhalb, sondern in den Kanon mit den deutschen Kollegen Schulter an Schulter hinein[schreibt].« (Rădulescu 2016: 64) Zwar gibt es etliche vergleichende Forschungsarbeiten innerhalb einer Nationalliteratur aus einem deutsch-türkischen, deutsch-arabischen, deutsch-baltischen etc. Blickwinkel (vgl. Hofmann/Patrut 2015), jedoch werden genuin komparatistische Verfahren vermisst. Dabei versprechen komparatistische Ansätze einen hohen Erkenntnisgrad im Sinne von >europäischer/n Migrationsliteratur(en)<, die das Phänomen polyperspektivisch innerhalb mehrerer sprachlich-kultureller Kontexte beleuchten und im Rahmen eines breit gefassten Migrationsbegriffs einen erheblichen Beitrag für eine literarische Selbstvergewisserung Europas leisten könnten. Da gerade Migrationsliteratur wie kaum ein anderes literarisches Phänomen die gesellschaftlichen Realitäten der partizipierenden kulturellen Entstehungs- und Handlungsorte literarisiert und somit wichtige Aufschlüsse über Erzähl- und Schreibstrategien der 
europäische(n) Migrationsliteratur(en) ${ }^{1}$ gewährt, bevorzuge ich den Begriff der >europäische(n) Migrationsliteratur(en)<, um auf vergleichende Synthesen auf der Textebene, die diese Literaturen zueinander in Beziehung setzen, und auf deren Spezifika, die sich auf unterschiedliche europäische Rahmenbedingungen beziehen, aufmerksam zu machen.

Den vorliegenden Beitrag verstehe ich infolgedessen insofern als >europäischく, als ich den deutschsprachigen Roman Sterne erben, Sterne fürben. Meine Ankunft in Wörtern von Marica Bodrožić (2007) und den griechischsprachigen Roman Man nennt mich Europa (2010) von Gazment Kaplani in Bezug auf gemeinsame Erzähl- und Schreibstrategien miteinander vergleiche. ${ }^{2}$ Trotz unterschiedlicher Entstehungsvoraussetzungen und Schreibintentionen habe ich mich für diese beiden Romane entschieden, da sie thematisch die Einwanderung in ein westliches Land und Reminiszenzen an die jeweiligen kommunistischen Lebenswelten behandeln. Meine These dabei ist, dass die Adaption der narrativen Thematik der zeitgenössischen Migration gemeinsame Erzähl- und Schreibstrategien zur Folge hat, die jenseits der Sprache, in der das jeweilige Werk geschrieben ist, einem >Gedächtnis europäischer Literatur ${ }^{3}$ Rechnung trägt.

\section{Faktoren aussertextlicher Kontexte}

Kurz zu den Autoren: Marica Bodrožić wurde 1973 in Dalmatien geboren und ist 1983 nach Deutschland übergesiedelt. Sie schreibt Gedichte, Romane, Erzählungen und Essays und hat zahlreiche Preise bekommen, wie den Kunstpreis Berlin (damals Förderungspreis) für Literatur der Akademie der Künste, den Literaturpreis der Europäischen Union oder den Literaturpreis der Konrad-Adenauer-Stiftung. Sie lebt als freie Schriftstellerin in Berlin.

Gazment Kaplani wurde 1967 in Albanien geboren und flüchtete nach dem Fall des Hotza-Regimes zu Fuß nach Griechenland. Er hat Philosophie an der Universität Athen studiert und in Politikwissenschaften promoviert. Kaplani hat einen Lyrikband auf Albanisch, Kontrabande Muze, herausgegeben und drei Romane auf Griechisch: Kleines Tagebuch der Grenzen (2006), Man nennt mich

1 | Eine komparatistische Ausnahme stellt die zuletzt erschienene Arbeit von Carmine Chiellino (vgl. 2016) dar, die jedoch für eine interkulturelle Literatur in deutscher Sprache ‘ plädiert. Ob aber der allgemein verstandene Begriff der ,Migrationsliteraturı durch interkulturelle Literatur in deutscher Spracher ersetzt werden kann, wäre eine lohnende weiterführende Forschungsarbeit.

2 I Im Weiteren werde ich mich auf folgende Ausgaben beziehen: Bodrožić 2016 (im Text nachfolgend ausgewiesen als Sterne) und Kam入ávı 2010 (im Text ausgewiesen als Europa). Die Übersetzung der griechischen Abschnitte aus Europa ins Deutsche von A.B.

3 | Zum interkulturellen Gedächtnis`, das sich auf europäische Literatur bezieht, siehe ausführlicher Chiellino 2016. 
Europa und Die letzte Seite (2012). Heute lebt der Autor in den USA (vgl. Alijai 2013: 71f.).

In Bodrožićs Roman Sterne pendelt der Plot stetig zwischen den Zeitebenen der Vergangenheit und der Gegenwart. Der Roman erzählt von der Kindheit der Ich-Erzählerin in Dalmatien und davon, wie sie mit dem Großvater aufwuchs, in ein hessisches Dorf übersiedelte und als Erwachsene nach Paris zog. In einem tagebuchartigen Gestus (vgl. Rădulescu 2013: 98) verweben sich »erzählte Lebenserinnerungen mit reflexiven, abstrahierenden, sentenzartigen Abschnitten« (ebd.: 99).

In Kaplanis Roman wiederum ist eine stetige Überschreitung der Zeitebenen festzustellen, da eine Pendelstruktur auf allen drei Ebenen zu vernehmen ist. Die Vergangenheit gehört den kommunistischen Zeiten in Albanien und der Einwanderung in Griechenland. Die Gegenwart wird durch 21 vom Autor real geführten Interviews dargestellt, die verschiedene Migrantenschicksale in Griechenland thematisieren und, indem sie monologisch als Selbstzeugnisse an verschiedenen Stellen des Plots positioniert sind, den Erzählfluss unterbrechen. Die Zukunft schließlich > gehört< einer fiktiven Gegenwart, denn die Handlung ist im Albanien des Jahres 2041 angesiedelt, als der Ich-Erzähler nach seiner langjährigen Emigration in Griechenland zurückkehrt. Albanien sei nun ein gelobtes Land, das Migranten und Flüchtlinge aus Asien und Afrika aufnimmt. Auch in diesem Roman alternieren Lebenserinnerungen mit reflexiven, sentenzartigen Abschnitten. Auf der Gegenwartsebene handelt es sich hauptsächlich um Erinnerungen an Dialoge des Ich-Erzählers mit seiner ersten Freundin in Griechenland namens Europa (ein konventioneller, wenn auch seltener griechischer Frauenname), wobei an verschiedenen Stellen die Mehrdeutigkeit des Wortes spielerisch eingesetzt wird.

\section{Gemeinsame Erzähl- Und Schreibstrategien}

Mit dieser knappen Beschreibung des Plots lässt sich bereits eine in beiden Werken ähnliche Erzählstruktur konstatieren, wobei hinzuzufügen ist, dass die Pendelstruktur zwischen dem Leben der Ich-Erzähler in der Fremde und der Beschreibung der Zeit der »Zugehörigkeit zur Herkunftskultur« (Chiellino 2016: 150) ein Charakteristikum ist, dass auch bei anderen Autoren der europäischen Migrationsliteraturen anzutreffen ist (vgl. ebd.), wie Salman Rushdies Satanic Verses (1988) und Artur Beckers Kino Muza (2003). Im Gegensatz zu anderen Autoren potenziert Kaplani jedoch diese Pendelstruktur durch die Aufsplitterung der Vergangenheit, da sich das Vertraute sowohl auf das Leben im kommunistischen Albanien als auch auf das Leben in Griechenland bezieht und darüber hinaus zusätzlich die Zukunft inszeniert wird.

Ein anderes gemeinsames Merkmal beider Romane ist das autofiktionale Erzählen. Obwohl bei Bodrožić die Ich-Erzählerin in der Mitte des Romans mit Eigennamen erscheint, heißt es gleich am Anfang des Romans: »Das Erzählen aus der Geschichte des menschlichen Herzens ist eine Befreiung aus der Umzäu- 
nung der Biographie« (Sterne 9). Bei Kaplani wird der Ich-Erzähler nicht beim Namen genannt, doch autobiographische Bezüge sind leicht erkennbar, zumal seine drei griechischsprachigen Romane als Trilogie verstanden werden können, die das Leben in Albanien, die Akkulturation in Griechenland und schließlich die Entscheidung, nach Amerika überzusiedeln, thematisieren. Die dreifache Pendelstruktur aber, die die erzählte Zeit auch in die Zukunft verlagert, unterminiert den autobiographischen Roman.

Hinzu kommt, dass - wie bei anderen Werken der Migrationsliteratur, etwa In deutschen Küchen von Franco Biondi (1997) - festzustellen ist, wie eng die etwaigen »Ich-Figurationen und ihre Wandlungen an die sprachliche Problematik gekoppelt sind « (Schenk 2016: 53). Die Tatsache, dass Autofiktionen aus der Sprache herrühren, wird bei beiden Autoren plastisch ausgemalt, da der Spracherwerb und Sprachgebrauch zu Ich-Verwandlungen führt. So heißt es bei Bodrožić:

In den Namen haben sich hin und wieder beweisbare Regungen der ersten Sprache erhalten. Filomena, ${ }^{4}$ beispielsweise, ist ein Wort, das sich bei mir wie ein Reisekoffer vor die Türen der deutschen Sprache gestellt hat. Das Wort wollte hier wohnen, auf der anderen Seite meiner selbst eine feste Sprachadresse haben, ansprechbar sein, gleich einem ferngereisten Verwandten, der nach der Kenntnis anderer Kontinente nun das Eigentliche erleben muss, sich selbst, als Mittler zwischen Vergangenheit und der eigenhändig gebauten Brücke zur Gegenwart. (Sterne 13f.; Hervorh. i.0.)

»[B]eweisbare Regungen der ersten Sprache« bestätigen Chiellinos Maxime »eines integrierten Lebenslaufs« (Chiellino 2016: 107), womit die interkulturelle Integration des Lebenslaufs des Ich-Erzählers gemeint ist. Es handelt sich, mit anderen Worten, um eine existentielle Notwendigkeit des Ich-Erzählers, Raumund Zeitkoordinaten, Vergangenheit und Gegenwart durch sprachliche Kontinuität in eine unverrückbare Nähe zu bringen (vgl. ebd.: 73). Dies wirkt sich m.E. auf die Struktur der Texte aus, da die interkulturelle Integration des Lebenslaufs die Erzählstrategie der Pendelstruktur unterstützt. Bezeichnenderweise betreffen die >Brücke < und die >Vermittlung < eine Art ästhetisches Programm, um zwischen >damals < und >jetzt<, zwischen >dort $<$ und >hier < weitgehend Kontingenz herzustellen, wobei sich die Pendelstruktur als Lebensprojekt entpuppt, »sich in beiden Kultur- und Sprachräumen alternierend« (ebd.: 70) zu entfalten. Bei Kaplani wiederum heißt es:

Ich glaube, dass ich angefangen habe in der griechischen Sprache zu wohnen, alsbald ich nicht mehr versuchte, für jedes Wort die albanische Äquivalenz zu finden. Oder, wenn ich sie suchte, dann passierte dies im Hintergrund des Denkens. In der griechischen Wirklichkeit habe ich angefangen zu wohnen, als ich aufhörte, Vergleiche zwi-

4 | Filomena war als Figur im Roman Das Gedächtnis der Libellen angedacht. Vgl. die Nachweise am Ende von Sterne. 
schen Hier und Dort anzustellen (Europa 339; Hervorh. i.0.). Den Sinn und die Gestalt einer Sprache gibt jedes Mal der Mensch, der sie spricht. (Ebd. 270)

Die Ich-Verwandlung betrifft die Entstehung des >Dritten Ortes $<$, da, losgelöst von sprachlichen und kulturellen Zwängen, das Leben in einem >Dritten Ort< ausgelebt wird. Der >Dritte Ort< ist nach Bhabha als diskursiver Rahmen zu verstehen, wo »dieselben Zeichen neu belegt, übersetzt, rehistorisiert und gelesen werden können « (Bhabha 2000: 57). Offensichtlich werden die sprachlichen und kulturellen Zeichen der Vergangenheit transformiert und tragen somit zu den Ich-Verwandlungen bei. In diesem Sinne ist auch die Verlagerung des fiktiven Albanien im Jahr $2041 \mathrm{zu}$ verstehen, da durch die Mehrfachkodierungen (vgl. Suppanz 2003: 27) desselben Diskurses eine Multiperspektivität entsteht, die kulturelle Transformationen inszeniert. ${ }^{5}$

Die Fluidität von Sinnzuschreibungen, die aus dem Dialog zwischen Vergangenheit und Gegenwart entspringt, drückt sich ebenso auf gattungsspezifischer Ebene aus, da beide Romane verschiedene Gattungen in sich vereinen. Bei Bodrožić handelt es sich um tagebuchartige realitätsnahe Aufzeichnungen, die eine reflexive Erzählhaltung umfassen, wobei die reflexive Erzählhaltung mit der starken Metaphorik alterniert. Durch den tagebuchartigen Gestus wird dem Leser der Eindruck vermittelt, dass ihn der Roman in die intimsten Erfahrungen des Ich-Erzählers einweihe und dies wiederum für den Ich-Erzähler eine kathartische Funktion auf dem Weg seiner Selbstfindung habe (vgl. Rădulescu 2013: 98). So übertritt der Roman durch den fiktiven Dialog die Schwelle zwischen Roman und Tagebuch, obgleich deutlich ist, dass die tagebuchartige Gattung inszeniert wird. Zusätzlich ist eine Mischung mit der Lyrik festzustellen, zumal der Plot mit einigen Gedichten ergänzt wird.

Bei Kaplani wird die Fiktion durch die real gehaltenen Interviews, die den Plot durchziehen, >gestört<. In beiden Werken werden also fließende Gattungsbegriffe favorisiert, um dadurch dem Gedanken an eine konstruierte Homogenität entgegenzusteuern. Gerade die Zerstörung vermeintlicher Homogenitäten, ob auf sprachlicher, erzähltechnischer oder gattungsspezifischer Ebene, ist ein weiteres wichtiges Merkmal europäischer Migrationsliteraturen, das aber immer im Hinblick auf einen sprachlich integrierten Lebenslauf zu sichten ist. ${ }^{6}$

5 | Ähnlich stellen Hofmann/Patrut (2015: 18) in Bezug auf den postkolonialen Diskurs heraus, dass die Literatur durch die verschiedenen Formen der Multiperspektivität die Notwendigkeit darlegt, "bornierte eurozentrische Perspektiven zu überwinden". 6 | Das Konstruierte der Homogenität ist, ohne die spezifische sprachliche Facette von Migrationsliteratur, selbstverständlich auch bei deutschen Autoren, wie z.B. Christoph Hein, anzutreffen. Vgl. für eine entsprechende Textanalyse Leskovec 2011: 130. 


\section{Gemeinsamkeiten auf inhaltlicher Ebene}

\subsection{Narratives Thema: Fiktionalisierung eines breiten Migrationsbegriffs}

Bezeichnenderweise bildet in beiden Romanen die Konfiguration eines breiten, dekonstruktiven Migrationsbegriffs einen thematischen Schwerpunkt. So beschreibt die Ich-Erzählerin im Prosaband von Bodrožić ihre Mutter als eine Frau, die nach Deutschland ausgewandert ist, um sich vornehmlich von gesellschaftlichen Zwängen zu befreien.

In Wirklichkeit war auch keiner von uns nur der Arbeit wegen in ein anderssprachiges Gebiet gegangen [...]. Sie [die Mutter; A.B.] ging fort, weil das Fortgehen ihr die einzige Möglichkeit bot, für etwas anderes als für die Tradition, für die Ehre, für Hab und Gut, für die Felder und die Sittengefühle inrer Familie zu leben. Sie war keine Gastarbeiterin. Sie ist eine geworden, weil man damals kein Wort für Frauen hatte, die sich als Frauen auf die Reise gemacht hatten; und nicht als Geldverdienerinnen. (Sterne 20)

Deutlicher könnte die Absage an einen überkommenen Migrationsbegriff bzw. Gastarbeiterbegriff nicht zum Ausdruck gebracht werden. Die Ich-Erzählerin wendet sich gegen essentialistische Festschreibungen, da als Essenz der Migration die Bewegung, die Auswanderung verstanden wird und Migration mehrere Gründe haben kann, die nicht im finanziellen Bereich zu verankern sind. Es wird demzufolge fiktiv ein breiter, dekonstruktiver Migrationsbegriff literarisiert, der auch auf der Handlungsebene die Multiperspektivität begünstigt und - vor allem - die Begrifflichkeit literarisch konfiguriert.

In Kaplanis Roman dominiert der thematische Aspekt der Migration und kann weitgehend von der im Werk aufscheinenden aufklärerischen Schreibintention erklärt werden, da, abgesehen von den 21 eingeflochtenen Interviews, explizite Neukodierungen der Migration auf fiktiver Ebene durchgespielt werden. So heißt es am Ende des Romans:

Migration ist ein Spiegel, der unser Gesicht wiederspiegelt [sic]. Wenn wir Migration als Gelegenheit betrachten, dann wird sie Gelegenheit. Wenn wir Migration als Gefahr betrachten, dann wird sie Gefahr. Weil der Andere nicht der Fremde, sondern Spiegel ist, in dem in Vergrößerung die Alpträume und die guten Eigenschaften des Eigenen projiziert werden... (Europa 346; Hervorh. i.0.).

Migration ist hier infolgedessen vollkommen von essentialistischen Sinnzuweisungen befreit, da Migration als privater und gesellschaftlicher Prozess dargestellt wird. Ungeachtet der fiktiven Umsetzung eines breiten Migrationsbegriffs stellt $m$.E. jedoch eine eigene »Schreibweise der Migrationsliteraturen« (Schenk 
2004: IX) ${ }^{7}$ die Tatsache dar, dass Begriffserklärungen zum Diskurs der Migration narrativ thematisiert werden. Dies wird noch deutlicher in folgendem Zitat Kaplanis: »Migration bedeutet, dein Leben von Null anzufangen. In einer Sprache zu erzählen, die nicht deine Muttersprache ist, ist, als ob du die Narration deines Lebens neu startest. Deswegen verstand ich das Griechische wie neue Schuhe, die mein Verlangen nach schnellem Laufen herausforderten.« (Europa 40)

Bei beiden Romanen werden in einem reflexiven Erzählmodus Begriffe zum Migrationsdiskurs explizit definiert. Es handelt sich dabei um eine besondere Schreibstrategie, die wenig von der entsprechenden Forschung beachtet worden ist, sich aber stark an spezifische »Schreibweisen der Migrationsliteraturen« anlehnt. Im Grunde genommen bestätigt sich auch in den zeitgenössischen Migrationsliteraturen das enge Zusammenspiel zwischen Fiktion und Realität, denn im Bekenntnischarakter der Narration werden sowohl die Auswirkungen der erlebten Migrationserfahrungen fiktionalisiert als auch auf reale Migrationskontexte Bezug genommen. Es ist kein Zufall, dass bei Bodrožić der Begriff des >Gastarbeiters < (ein genuin deutsches Phänomen) als homogenisierend und sprachlich defizitär ausgewiesen und bei Kaplani der Begriff der >Migration< mit der griechischen Sprache verwoben wird.

Infolgedessen können m.E. drei wesentliche Merkmale der Migrationsliteraturen festgehalten werden: erstens der Rekurs auf den jeweiligen, real erlebten Migrationsdiskurs, was die Verwendung von Migrationsliteraturen im Plural legitimiert, zumal dadurch die Kulturgebundenheit jedes literarischen Textes zum Ausdruck gebracht wird; zweitens die explizite Inszenierung eines breiten Migrationsbegriffs, der die Fluidität und das Konstruierte der Homogenität als inhaltliches und strukturelles Merkmal umsetzt; und drittens die emanzipatorische Schreibhaltung, die den >Migrationsbegriff< zu emanzipieren trachtet, indem er nicht ausschließlich im Kontext von finanzieller Armut verortet wird. Darüber hinaus wird der aufklärerische Impetus als Schreibintention durch die erlebte Innensicht der Migrationserfahrung im autofiktionalen Erzählen deutlich. In den hier behandelten Werken wird Letzteres durch die reflexiven Passagen erreicht. Mit anderen Worten diktiert die Thematik der zeitgenössischen Migration spezifische Charakteristika in der narrativen und inhaltlichen Konsistenz der jeweiligen Literaturen.

\subsection{Narratives Thema: Kommunismus}

Reminiszenzen an den Kommunismus fallen in Bodrožić' Roman eher rar aus, da der Fokus auf der kathartischen Funktion der deutschen Sprache liegt, die zusammen mit der ersten Muttersprache ${ }^{8}$ Kindheitstraumata zu eliminieren vermag:

7 | Die Bezeichnung lehnt sich an Schenk an, ohne sich jedoch ausschließlich auf die deutschsprachige Literatur zu begrenzen.

8 | Das Serbokroatische und das Deutsche werden entsprechend als erste und zweite Muttersprache bezeichnet, was die Verschwisterung beider für die Subjektkonstitu- 
1983 hatte ich als neunjähriges Kind, zusammen mit meinen Geschwistern, das Land verlassen, hatte bis dahin an jeder Maiparade teilgenommen und die Pioniermütze getragen wie alle anderen Kinder auch. [...] Es war üblich, die Lehrer mit "Genosse" anzusprechen. Das sollte unsere Brüderlichkeit ausdrücken. [...]

Einer der Gründe, weshalb die sozialistische Gleichheitsidee in der Realität gescheitert ist, war mit der Vorstellung der Machthaber verknüpft, Menschen eine Sprache zu verordnen. [...] Wir litten an Dingen, an denen alle Kinder leiden: ich an der Abwesenheit meiner Eltern, an der Armut und meiner eigenen Ungeschicktheit, die mich immer von den anderen trennte und als einen typischen Verlierer-Fall unserer Familie darstellte. (Sterne 63).

Anschaulich wird an dieser Stelle der erlebte Alltag mit den eigenen existentiellen Nöten in Verbindung gebracht, was zwar nicht über die Prägung durch das Regime im Kindesalter hinwegtäuscht, aber über den Erkundungscharakter des eigenen Schreibens die Spuren des interkulturellen Gedächtnisses aufrollt. Es bestätigt sich durch Bodrožić' Werk, dass dessen Sprache nicht nur Trägerin des kulturhistorischen Gedächtnisses ist (vgl. Chiellino 2016: 48), sondern auf der Folie des Zusammenspiels der Sprachen die Integration der Lebensabschnitte der Protagonistin (vgl. ebd.: 119) intendiert wird. Dies hat wie bei anderen Autoren, z.B. Andrea Franzettis Erzählung Der Großvater (1985), die Konsequenz, dass zwischen den zwei Sprachen des Ich-Erzählers »ein intensiver Austausch an Informationen stattfindet, aus dem sich die deutsche Sprache des Werkes ernährt, um weiter erzählen zu können.« (Ebd.) Aus Bodrožić' Zitaten geht hervor, dass sowohl sprachliche Segmente wie >Genosse < und >Brüderlichkeit< als auch soziohistorische Gegebenheiten im sozialistischen Alltag >ins Deutsche< übersetzt werden, um das sprachliche Kontinuum des interkulturellen Gedächtnisses herzustellen.

Die Tatsache, dass es im Fall der Migrationsliteraturen kein Kontinuum zwischen der Sprache und dem soziohistorischen Wissen des Autors und des Lesers gibt, wird ebenso aus folgendem Zitat Kaplanis deutlich:

So sprach ich zu Europa über all meine lächerlichen und traumatischen Erfahrungen von den Paraden in Albanien. Dass man uns spezifische Übungen für die Feiern der Partei lernen ließ, für den Geburtstag des großen Staatsmannes, für die Maiparade, für den Tag der nationalen Unabhängigkeit. Sowieso ähnelten wir einer Kaserne mit Soldaten, die sich ständig auf Paraden vorbereitete. (Europa 70)

Die explizite Ansprache an die Freundin namens Europa - unverkennbar an dieser Stelle der mehrdeutige Gebrauch des Namens, da der Ich-Erzähler zu Europa spricht - verdeutlicht, dass dem griechischen Lesepublikum, obgleich in griechischer Sprache erzählt wird, zusätzliches soziohistorisches Wissen vermittelt werden muss. Folglich wird der künstlerische Einsatz der Sprache, die eine ex-

tion vergegenwärtigt, z.B.: "Aber Erinnerung verwaltet sich noch einmal anders in einer zweiten Muttersprache." (Sterne 140). 
plizit erklärende Funktion einnimmt, mit der Vermittlung für das griechische Lesepublikum >fremder < soziohistorischer Gegebenheiten in Einklang gebracht. Dadurch werden aber nicht nur Erlebnisse des Fremden in Form von künstlerischer Produktivität ${ }^{9}$ inszeniert, sondern ebenso Erlebnisse des Eigenen in der Fremdsprache. Demzufolge zeigt sich, dass über das narrative Erklären hinaus spezifische Informationen vermittelt werden, die das interkulturelle Gedächtnis konstituieren. Die erklärende Schreibstrategie ist zusätzlich auf die aufklärerische Erzählintention Kaplanis zurückzuführen, wodurch die im Vergleich zu Bodrožić häufigeren, aber insgesamt gering gehaltenen, thematischen Bezüge zum Leben im kommunistischen Albanien gedeutet werden können.

\subsection{Narratives Thema: Balkan}

Der Balkan nimmt nach Previŝić eine topographische und historische Zwischenposition ein. »[D]er Balkan wird bis weit ins 19. Jahrhundert hinein als >europäische Türkei< bezeichnet, als Landbrücke zwischen >Mitteleuropa < und dem >Nahen Osten < halb christlich, halb moslemisch, sozusagen ein Zwitter, zerrissen zwischen einem >byzantinisch-osmanischen < Erbe und einer europäischen Orientierung « (Previŝić 2010: 191). Auch wenn man dieser leicht ironisierenden Begriffsbestimmung nicht beipflichtet, so kann trotzdem festgehalten werden, dass der Balkandiskurs vornehmlich eine Zwitterfiguration verkörpert. Bei Bodrožić nimmt die Inszenierung des Balkandiskurses, im Gegensatz zu Kaplani, einen überschaubaren Raum ein. Der >Balkan< kommt nur an einer Stelle vor, als sich die Ich-Erzählerin in Mile, einen bosnischen Kroaten, und ihre Schwester in Rašo, einen kroatischen Serben, verliebt. »Rašo spielte auf der Gitarre das jugoslawische Kultlied >Balkane moj<, Mein Balkan, wir saßen im Park herum, bis tief in die Nacht, rauchten die Zigarettenpäckchen leer und erklärten unseren neuen Freunden die deutsche Sprache, ließen das $r$ rollen, vor und zurück.« (Sterne 27f.; Hervorh. i.O.)

Das Balkanlied hat an dieser Stelle insofern eine einschließende Funktion, als es das gemeinsame Gedächtnis aller Ex-Jugoslawen zum Vorschein bringt und somit die ethnischen Grenzziehungen in Folge des Krieges ad absurdum führt. Eines der zentralen Anliegen des Romans ist es, auf den Irrsinn der Zersplitterung Ex-Jugoslawiens nach ethnischen Kriterien aufmerksam zu machen und durch die Literarisierung mehrerer erlebter Beispiele den Zusammenhalt aller Auslandsjugoslawen in Europa zu vermitteln. Auch wenn die Ich-Erzählerin von »jugoslawischer Weise« (ebd.: 33) spricht, deren Quintessenz sie nicht erklären kann, so bleibt, dass, auch wenn sich die Völker des ehemaligen Jugoslawien »zu Hause stritten, bekriegten und ermordeten, sie im Ausland unzer-

9 | Interessanterweise stellt Schickhaus zum Werk Yoko Tawadas heraus, dass die "Lokalisierung des Fremden außerhalb der Gesellschaft nicht mehr zum Kennzeichen oder Manko einer Gruppe von Migranten gilt, sondern zur Voraussetzung künstlerischer Produktivität، erhoben wird." (Schickhaus 2016: 89) 
trennlich [waren], hörten die Chansons des einen Volkes, die Lieder des anderen, und alles wurde wieder eins.« (Ebd.: 34)

Durch Bodrožić' Roman scheint sich das schriftstellerisch interkulturelle Desiderat einzulösen, welches »das politisch prekäre Verhältnis nicht nur zwischen den Staaten des ehemaligen Jugoslawiens, sondern auch zur EU entschärfen helfen « (Previsić́ 2009: 189f.) kann, zumal über die fiktiven Beispiele der erfahrbaren, aber nicht näher zu definierenden »jugoslawischen Weise« der Ex-Jugoslawen im Ausland Trennungen nach Kriterien der Nationenbildung als obsolet erklärt werden. »Jetzt erst [in Deutschland; A.B.] begriff ich, dass ich offiziell eine Kroatin bin, die in europäischen Städten noch immer allen möglichen Jugoslawen hinterhergeht, um ihre Stimme zu hören [...] ganz gleich, ob in Paris, Lissabon, Frankfurt oder Berlin.«(Sterne 72)

Aus dem Zitat geht hervor, weswegen der Balkan im Roman keine identitätsstiftende Funktion hat: Diese übernimmt das nicht näher definierbare >Jugoslawische $<$. Europa wirkt dabei wie eine Kulisse. Obwohl aber Balkan- und Europadiskurse nur in geringem Ausmaß durchgespielt werden, wird durch die, für deutsche Leser unbekannten, transportierten sozialgeschichtlichen Gegebenheiten der Ex-Jugoslawen im Ausland ein Gedächtnis europäischer Literatur in deutscher Sprache intendiert.

In Kaplanis Roman wiederum gibt es viele Bezüge zum Balkandiskurs, der identitätsstiftend eingesetzt wird. An dieser Stelle kann nur exemplarisch der Balkan-Katalog angeführt werden, den der Ich-Erzähler anfertigt, um die >balkanische Art< zu beschreiben:

Als ich später auf dem Balkan reiste, ohne Europa, leider, hatte ich einen BalkanKatalog angefertigt, den ich bei jeder Reise bereicherte. Du verstehst, dass du dich auf dem Balkan befindest, wenn du siehst, dass die Moslems viel trinken. [...] Du verstehst, dass du dich auf dem Balkan befindest, wenn du siehst, dass das magische Getränk Raki oder Rakĭ heißt. Außer ein Getränk zu sein, heilt der Raki alle Krankheiten, von Schnupfen bis zur Arthritis. [...] Du verstehst, dass du dich auf dem Balkan befindest, wenn dir ein schulisches Geschichtsbuch als von der militärischen Zentralstelle geschrieben scheint. [...] Du befindest dich auf dem Balkan, wenn sich immer jemand findet, der dir dessen Tür und Seele öffnet, bevor er deinen Namen kennt. [...] Du verstehst, dass du dich auf dem Balkan befindest, wenn du die Interviews mit den wichtigen Staatsleuten in der Cafeteria durchführst. Weil der Balkan ein großes Dorf ist, das sich anstrengt Paris und London nachzuahmen. (Europa 309)

Die anfangs zitierte Definition des Balkans von Previŝić scheint im obigen Zitat eine deckungsgleiche Entsprechung zu finden. Der Balkan ist der identitätsstiftende Raum des Disparaten. Abgesehen von der Wahrhaftigkeit dieses Katalogs, ist nicht zu übersehen, dass dadurch Stereotype gefestigt werden. Die Tatsache aber, dass Holismen trotzdem mit Skepsis begegnet wird, kann vom Bedauern der Abwesenheit der Freundin Europa, die als Verkörperung des Fremden gegenüber dem Ich-Erzähler eine kritische Instanz darstellt, sowie vom >Dialog der Gattungen $<$ abgelesen werden. In einem nachstehenden Interview bekundet der 
Interviewte: »Wo immer ich auch gereist bin, meine besten Gesellen waren immer Menschen aus dem Balkan, vornehmlich Griechen. So ist in mir ein anderes Stereotyp entstanden, dass nämlich wir Balkanier etwas anderes sind als die Westler. Es ist mein letztes Stereotyp, leider...« (ebd.: 314). Das Bedauern im Interview könnte als zusätzlicher Hinweis darauf verstanden werden, dass auf die Fiktion Bezug genommen wird.

Fazit: Der Balkan kann als Europa in Europa aufgefasst werden, der sich, trotz seiner Zwittergestalt, identitätsstiftend auswirkt und eine andere Sorte von Europäern, eine Art >Mimikry-Europäer<, zum Vorschein bringt. Der Konstruktionscharakter des Balkans wird jedoch in beiden Romanen wenig angetastet. Sogar bei Bodrožić schwingt die Vorstellung mit, dass die >jugoslawische Weiseく auf die Lebenswelt des Balkans zurückzuführen ist. Es wäre sicherlich von großem Interesse, andere >balkanische < Migrationsliteraturen heranzuziehen, um obige Befunde zu erkunden. Die Tatsache, dass bei Bodrožić die identitätsstiftende Funktion nicht dem Balkan, sondern einer nicht näher definierten jugoslawischen Substanz zugewiesen wird, kann mit den Konsequenzen des Krieges und dem Widerstand gegenüber dem Ideologem der ethnischen Säuberungen erklärt werden. Auch wenn Europadiskurse in beiden Romanen kaum behandelt werden, so ist ihr Vorhandensein als künstlerischer Referenzpunkt nicht zu unterschätzen, da durch sie lokale soziohistorische Gegebenheiten in die Sprache des europäischen Ziellandes infiltriert werden und sie einen wesentlichen Beitrag zur Entstehung eines >Gedächtnisses europäischer Literatur< leisten.

\section{Schlussbetrachtungen}

Die Performanz des Eigenen und des Fremden im Netzwerk der sprachlichen, strukturellen und inhaltlichen Dialogizität macht zweifelsohne die besonderen »Schreibweisen der Migrationsliteraturen« aus. Die Komplexität des modernen Migrationsbegriffs, der in derartigen Literaturen konfiguriert wird, wirkt sich auf allen Schreibebenen aus, sodass durchaus von europäischen Migrationsliteraturen, die jenseits der gewählten Sprache ein migrantisches Schreiben performen, die Rede sein kann. Die hier vorgeführten Textanalysen haben gezeigt, dass über den sprachlich-kulturellen Bezugsrahmen und der Schreibintention hinaus viele Gemeinsamkeiten konstatiert werden konnten. Das gemeinsame migrantische Schreiben findet seinen Niederschlag vorbildhaft in der Kongruenz von Form und Inhalt, indem ein Migrationsbegriff, der auf Bewegung ausgerichtet ist und eine emanzipatorische Wirkung intendiert, durch die Wahl einer entsprechenden Konstruktion auf der Strukturebene adaptiert wird. Schließlich stellen Migrationsliteraturen eine große Herausforderung dar, sowohl für die Nationalliteraturen als auch für die Komparatistik. Denn einerseits handelt es sich bei diesem literarischen Phänomen um eine Literatur mit doppelter Staatsbürgerschaft (vgl. Blioumi 1999: 355f.), die die jeweilige Nationalliteratur erneuert, zumal erlebte geschichtliche Ereignisse eines anderen Landes in der jeweiligen Nationalliteratur aufgenommen werden. Und anderseits ist zu diskutieren, 
ob, holistisch betrachtet, von einer >europäischen Migrationsliteratur < die Rede sein kann, die ein >Gedächtnis europäischer Literatur $<z u$ inszenieren vermag.

\section{LITERATUR}

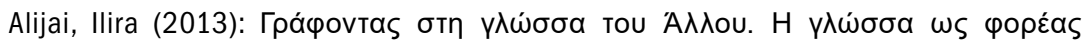

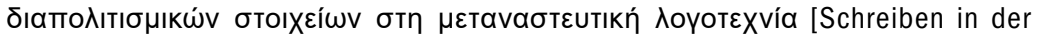
Sprache des Anderen. Die Sprache als Träger interkultureller Elemente in der Migrationsliteratur]. Masterarbeit. Panteion Universität Athen.

Bhabha, Homi K. (2000): Die Verortung der Kultur. Mit einem Vorwort v. Elisabeth Bronfen. Aus dem Engl. v. Michael Schiffmann u. Jürgen Freudl. Tübingen.

Blioumi, Aglaia (1999): Migrationsliteratur, der schwarze Peter für die Komparatistik? Plädoyer für eine Komparatistik mit doppelter Staatsbürgerschaft. In: arcadia 34, H. 2, S. 355-365.

Bodrožić, Marika (2016): Sterne erben, Sterne färben. Meine Ankunft in Wörtern. München.

Chiellino, Carmine (2016): Interkulturelle Literatur in deutscher Sprache. Das große $A B C$ für interkulturelle Leser. Bern.

Hofmann, Michael/ Patrut, Iulia-Karin (2015): Einführung in die interkulturelle Literatur. Darmstadt.

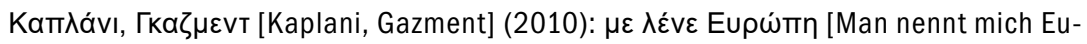
ropa]. Athen.

Krauze-Olejniczak, Alicja (2016): Migrationsroman, der gar keiner sei: Malin Schwerdtfegers Cafe Saratoga. In: Christel Baltes-Löhr/Raluca Rădulescu (Hg.): Pluralität als Existenzmuster. Interdisziplinäre Perspektiven auf die deutschsprachige Migrationsliteratur. Bielefeld, S. 97-116.

Leskovec, Andrea (2011): Einführung in die interkulturelle Literaturwissenschaft. Darmstadt.

Previŝić, Boris (2009): Poetik der Marginalität: Balkan Turn gefällig? In: Helmut Schmitz (Hg.): Von der nationalen zur internationalen Literatur. Transkulturelle deutschsprachige Literatur und Kultur im Zeitalter globaler Migration. Amsterdam/ New York, S. $189-203$.

Ders. (2010): Zwischen Diskursivität und Faktualität: Interkulturalität und literarische Imagination auf dem balkanischen Prüfstand des jugoslawischen Zerfalls. In: Dieter Heimböckel/Irmgard Honnef-Becker/Georg Mein/Heinz Sieburg (Hg.): Zwischen Provokation und Usurpation. Interkulturalität als (un)vollendetes Projekt der Literatur- und Sprachwissenschaften. München, S. 191-203.

Rădulescu, Raluca (2013): Die Fremde als Ort der Begegnung. Untersuchungen zu deutschsprachigen südosteuropäischen Autoren mit Migrationshintergrund. Konstanz.

Dies. (2016): Die Lyrik José F.A. Olivers. Versuch einer "modernen « interkulturellen Hermeneutik. In: Dies. / Christel Baltes-Löhr (Hg.): Pluralität als Existenzmuster. Interdisziplinäre Perspektiven auf die deutschsprachige Migrationsliteratur. Bielefeld, S. 63-78. 
Schenk, Klaus (2004): Vorwort. In: Ders. (Hg.): Migrationsliteratur. Schreibweisen einer interkulturellen Moderne. Tübingen, S. VII-XI.

Ders. (2016): Formen des Ich-Erzählens in der inter-/transkulturellen Literatur. In: Christel Baltes-Löhr/Raluca Rădulescu (Hg.): Pluralität als Existenzmuster. Interdisziplinäre Perspektiven auf die deutschsprachige Migrationsliteratur. Bielefeld, S. 47-62.

Schickhaus, Tobias Akira (2016): Polypolare Über-setzungen. Eine historiographische Lokalisierung der Chamisso-Literatur am Beispiel Yoko Tawadas. In: Christel BaltesLöhr/Raluca Rădulescu (Hg.): Pluralität als Existenzmuster. Interdisziplinäre Perspektiven auf die deutschsprachige Migrationsliteratur. Bielefeld, S. 79-96.

Suppanz, Werner (2003): Transfer, Zirkulation, Blockierung. Überlegungen zum kulturellen Transfer als Überschreiten signifikatorischer Grenzen. In: Federico Celestini / Helga Mitterbauer (Hg.): Ver-rückte Kulturen. Zur Dynamik kultureller Transfers. Tübingen, S. 21-33. 
